\title{
$\$$ Research Square

\section{Development and Evaluation of a New Assessment Tool for Myelopathy Hand Using Virtual Reality}

\section{Xin-Jin Su}

Shanghai Jiao Tong University School of Medicine

\section{Zheng-Dong Lv}

Shanghai Jiao Tong University School of Medicine

\section{Wei-Zhong Zhang}

Shanghai Jiao Tong University School of Medicine

\section{Cang-Long Hou}

Changhai Hospital

\section{Quan Li}

Shanghai Jiao Tong University School of Medicine

Shen Hong-Xing ( $\nabla_{\text {jizhuwai@126.com ) }}$

Shanghai Jiao Tong University School of Medicine Affiliated Renji Hospital

\section{Research article}

Keywords: Myelopathy-hand Functional Evaluation System, Validity, Reliability, Effectiveness, Myelopathy hand.

Posted Date: August 5th, 2020

DOI: https://doi.org/10.21203/rs.3.rs-53380/v1

License: (c) (i) This work is licensed under a Creative Commons Attribution 4.0 International License.

Read Full License 


\section{Abstract}

Background: Impairment of hand function in myelopathy patients is commonly observed. However, no objective and effective method is widely accepted in clinical practice.

Objective: To evaluate the validity, reliability and effectiveness of a new assessment tool, Myelopathyhand Functional Evaluation System (MFES), in evaluating the hand function in the 10-s hand grip-andrelease test (10s G-R test).

Methods: MFES is mainly composed of a pair of wise-gloves and a computer with software. All included patients received optimal surgery treatment. The Japanese Orthopedic Association (JOA) scores were performed and the hand function was evaluated using MFES before operation, and 6 months after surgery. All patients were asked to perform the 10 s G-R test and the hand movements were simulated and converted into waveforms by MFES. The waveform parameters were measured and analyzed. The validity and test-retest reliability were assessed.

Results: The Bland-Altman showed significant agreement with a low bias and narrow limits of agreement. The test-retest reliability was high with a significant value $(r=0.911)$. The JOA scores and the number of G-R cycles significantly in postoperative increased. Correspondingly, the waveforms of ulnar three fingers were significantly higher and narrower, along with the significantly declined average time per cycle in postoperative. The number of the first five-second segment (N1) was significantly higher than the secondfive segment (N2) in postoperative, indicating the recovery of spinal cord function after surgery. The preoperative number of cycles was positively correlated with the improvement rates of JOA scores. And the increased number of cycles was significantly correlated with the improvement rates of JOA scores.

Conclusion: MFES is a reliable and valid assessment tool for myelopathy hand, which can detect small changes of neurological function after decompression surgery.

\section{Introduction}

Cervical spondylotic myelopathy (CSM), a common chronic progressive spinal disease, is mainly caused by spinal cord compression secondary to the degenerative changes of intervertebral discs or bone and soft tissue around, presented with hyperreflexia, loss of dexterity, and strength [1]. Impairment of hand function in patients with CSM is commonly observed, characterized by loss of strength and inability to grip and release rapidly with the fingers $[2,3]$. The hand dysfunction is also termed as myelopathy hand. Despite its high incidence, little is known about the underlying neuromechanical deficits. In a recent study, Smith et al. [4] believed that hyperreflexia and proprioceptive deficits were likely the primary drivers of hand dysfunction. The clinical significance of myelopathy hand is considerable [3], as the hand function was reported to be positively correlated with the severity of myelopathy, and the early improvement of postoperative hand function was commonly regarded as one of the objective measurements to reflect the efficacy of decompression surgery [3]. In summary, accurate assessment of hand function is helpful for 
the diagnosis of myelopathy and the formulation of optimal treatment, as well as for the evaluation of the efficacy of decompression surgery.

Due to its clinical significance, several symptom-based grading scales and performance tests had been developed in order to objectively and quantitatively assess the impaired hand function or predict the clinical outcomes of decompression surgery [2, 3, 5-15]. However, these are commonly complained of low sensitivity or low practicability for clinical use. Currently, no objective and effective evaluation method is widely accepted in clinical practice. Hence, our team designed Myelopathy-hand Functional Evaluation System (MFES), combining virtual reality technology with artificial intelligence. It offers possibilities of quantitative and automatic measurement of the hand motor dysfunction. In this study, we aimed to preliminarily evaluate its validity, reliability and effectiveness, serving as the first step toward the development of this new assessment tool.

\section{Methods}

\section{Myelopathy-hand Functional Evaluation System (MFES)}

The MFES is mainly composed of a pair of wise-gloves, a light, a computer with software, and two mutually perpendicular cameras (Fig. 1A). The wise-gloves were used to acquire and record dynamic signals in the course of metacarpophalangeal joint movement. It could convert analog signals to digital signals, and then transmit them to the software (Fig. 1B). Ultimately, the motor movements of the fingers were converted into waveforms by MFES. In addition, the MFES can automatically count the number of G$\mathrm{R}$ cycles, and calculate the average time per cycle and support playback. The hand motor movements were recorded by two cameras in real-time from front and side directions and showed them on the software, as references for subsequent comparison. The software interface mainly contains three regions: Waveforms; Basic information; Real-time video (Fig. 1C). It was compiled by Microsoft objectoriented visible integrated programming system-Visual C++. The detailed design of MFES was submitted as supplementary material.

\section{Study Design and Participants}

This is a prospective study. One hundred and thirty patients with cervical myelopathy ( 75 men and 55 women) were included in this study. The age of patients ranged from 34-83 years (mean age:

$59.03 \pm 10.18$ ). All patients received detailed information of this study and gave written informed consent. This study was approved by the Renji Hospital Ethics Committee. The demographic information of included patients was shown in Table 1, and the inclusion/exclusion criteria were as follows: Inclusion criteria: (1) Patients diagnosed as CSM with hand motor dysfunction as the chief complaint or presented with clinical symptoms of cervical myelopathy in the upper limbs and mechanical compression of cervical spinal cord identified by Magnetic Resonance Imaging; (2) Conservative treatment was ineffective for more than three months; (3) Age from 18 to 90 years; (4) Able to give informed consent; (5) Males and females; Exclusion criteria: (1) Patients with cervical myelopathy but had received related surgical treatments; (2) CSM patients only presented with sensation dysfunction but without complaints 
of hand motor dysfunction; (3) Patients with other causes of weakness of the hand, such as brain paralysis, rheumatoid arthritis (RA), congenital malformation, tumor, cervical spine trauma and so on; (4) Patients with other causes of weakness of grip which may be associated with myelopathy, such as C8 radiculopathy; (5) Patients only presented with lower limb symptoms.

\section{Surgery Treatment and Postoperative Management}

All patients were treated with Anterior Cervical Discectomy and Fusion (ACDF), Anterior Cervical Corpectomy and Fusion (ACCF), Posterior Cervical Laminoplasty (Laminoplasty) or Posterior Cervical Laminectomy and Fusion (Laminectomy), which were operated by different teams at the same center. Neurotrophic therapy was performed after surgery, and each patient was required to wear a cervical collar for one month after surgery.

\section{Japanese Orthopedics Association (JOA) Scores}

As an appropriate reference measurement, the severity of CSM was evaluated by Japanese Orthopedic Association scoring (JOA) system before operation, and 6 months after surgery [8]. Improvement rate of JOA score $=($ preoperative scores - postoperative scores) $/$ (17-preoperative scores) $\times 100 \%$ [14], which was divided into four levels: excellent ( $\geq 75 \%)$; good (50-74\%); general (25-50\%); and poor ( $₫ 25 \%)$.

\section{Testing Process}

Before starting the measurement, each patient was given a brief instruction of the testing procedures, and the operator needed to input basic information for each patient and assisted them wearing gloves. Then, with left palm pronated, each patient was asked to fully grip and release their fingers as rapidly as possible. The same process was repeated on the right hand (Fig. 1D). At the day before operation, all patients performed two tests, with a 1-h interval: (1) 10-s test to assess validity using Bland-Altman method; (2) 10-s test to assess test-retest reliability using Pearson correlation coefficient analysis. Three observers independently counted and analyzed the number of cycles over 10 s through the first 10-s video recorded by the two cameras. The average mean of the three was compared with that automatically counted by MFES to evaluate the validity, using Bland-Altman method. And test-retest reliability was assessed by comparing the two 10-s tests using Pearson correlation coefficient analysis.

\section{Measurement of Waveform Parameters}

The following waveform parameters were measured: Wave height ( $a, 0 \leq a \leq 20 \mathrm{~mm})$; Wave width (b囚0mm); The ratio of wave height to wave width (a/b). (Fig. 2)

\section{Statistical Analysis}

The paired $T$ test (SPSS 24.0) was used to evaluate the differences of the number of G-R cycles, the average time per cycle, the waveform parameters between preoperative and postoperative. The two-way mixed Intraclass Correlation Coefficient (ICC) was used to assess the inter-rater reliability of the three 
video observers. The validity of the MFES in capturing the number of G-R cycles was assessed using the Bland-Altman method, with human observation of the first 10-s G-R test as the reference measurement. The test-retest reliability of MFES in capturing the number of G-R cycles was evaluated using Spearman correlation coefficient analyses. IBM SPSS Statistics (Version 24.0) and Graphpad Prism (Version 8.0) were used to analyze all data. A $P$ value less than 0.05 was considered statistically significant $\left({ }^{*} P<0.05\right.$, $\left.{ }^{*} P<0.01, * * * P<0.001\right)$.

\section{Results}

The mean number of G-R cycles counted by three observers from the first video recordings was $13.93 \pm 4.20$. The average preoperative number of G-R cycles automatically counted by MFES was $13.58 \pm 4.31$ in the first 10 -s test, and $13.38 \pm 4.10$ in the second test.

\section{Inter-rater reliability, validity and reliability.}

The interclass correlation coefficient (ICC) showing agreement between three observers was high: ICC = $0.996(95 \% \mathrm{Cl}=0.994-0.998, P \otimes 0.0001)$. The correlation between the mean number of G-R cycles automatically counted by MFES and the mean number of G-R cycles counted by human observers were shown in Fig. $3 A(r=0.967, P \otimes 0.0001)$. The Bland-Altman plot (Fig. 3B) showed a bias of 0.354 cycles (SD $=1.070)$. The upper and lower limits of agreement were 2.451 and -1.744 cycles. Test-retest reliability at a 1 -h interval was performed, which indicated a significant value $(r=0.911, P \otimes 0.0001)$ in Spearman ranking correlation coefficient (Fig. 3C).

\section{Comparison of the preoperative and postoperative JOA scores.}

The mean JOA score significantly increased from $10.48 \pm 1.51$ to $13.22 \pm 1.89$ after decompression surgery $(P \otimes 0.0001)$. The mean improvement rates of JOA score was $45.01 \% \pm 17.04 \%$. The excellent and good rate reached $47.69 \%, 12$ of which were excellent, 50 of which were good, 59 of which were general, and 9 of which were poor.

\section{Comparison of the preoperative and postoperative number of cycles.}

The average number of G-R cycles significantly increased from $13.59 \pm 4.30$ to $16.00 \pm 4.92$ after surgery treatment $(P=0.0009)$. Correspondingly, the frequency of waveforms in postoperative was faster, and the bottom of the wave shapes was narrower than those in preoperative (Fig. 4A). There were significant differences of the number of cycles between the first five-segment (N1) and the second one (N2) in postoperative $(P=0.0002)$, but not in preoperative $(P=0.074)$, which could be directly observed through the waveforms (Fig. 4B.C).

\section{Correlation analysis between the number of cycles and the JOA scores.}

Correlation analysis between the number of cycles in preoperative and the improvement rates were performed, which indicated a significant value $(r=0.628, P \otimes 0.0001)$ in Spearman ranking correlation 
coefficient (Fig. 4D). Significant positive correlation between the increased number of cycles and the improvement rates of JOA scores was found ( $r=0.585, P \otimes 0.0001$, Fig. $4 E)$.

\section{Comparison of the waveform parameters in preoperative and postoperative.}

The wave shapes of the ulnar three fingers in postoperative were significantly higher $\left(P_{1} \otimes 0.0001, P_{4} \square\right.$ $\left.0.0001, P_{7}=0.0088\right)$ and narrower $\left(P_{2} \varangle 0.0001, P_{5} \rrbracket 0.0001, P_{8} \bowtie 0.0001\right)$ than those in preoperative (Table 2 , Fig. 4A). The waveforms of the thumb and index fingers in postoperative were significantly narrower $\left(P_{11}\right.$ $\left.0.0001, P_{14} \otimes 0.0001\right)$ than those in preoperative, but not higher $\left(P_{10}=0.553, P_{13}=0.762\right)$. The ratio $(\mathrm{a} / \mathrm{b})$ of five fingers were significantly higher in postoperative than that in preoperative $\left(P_{3} \varangle 0.0001, P_{6} \varangle 0.0001, P_{9} \square\right.$ $0.0001, P_{12} \otimes 0.0001, P_{15} \bowtie 0.0001$, Table 2). Correspondingly, there were significant declines of the average time per cycle in postoperative $(0.74 \pm 0.12 \mathrm{~s})$ than that in preoperative $(0.61 \pm 0.14 \mathrm{~s})(P \otimes 0.001)$. The average time per cycle could be directly calculated by MFES (Fig. 5).

\section{Discussions}

The purpose of this study was to preliminarily evaluate the validity, reliability and effectiveness of MFES in evaluating the hand function of myelopathy patients in the $10 \mathrm{~s}$ G-R test. And the results showed that MFES was valid, reliable and effective in the 10 s G-R test.

In this study, the high ICC and $95 \% \mathrm{Cl}$ values were consistent with the previous studies $[2,16]$, indicating that there was no significant discrepancy between three independent observers in counting the number of G-R cycles, and proving again that video observation is an appropriate reference measurement for this validation [17].

Compared with human observation, the MFES showed good consistency in counting the number of G-R cycles over $10 \mathrm{~s}$, with a significant value $(r=0.967)$. The Bland-Altman plot showed a bias of 0.354 cycles $(S D=1.070)$, indicating that the MFES tended to underestimate the number of cycles with an observation deviation of 0.354 and 0.462 cycles. This means that just $2.54 \%$ measurement errors in contrast to human observations. The incomplete and nonstandard G-R cycle may be counted by observers, but not by MFES, which may account for this result. The uniform distribution of data points across the BlandAltman scatter plot indicated no systematic difference across the range of number of cycles. Furthermore, the $95 \%$ limits of agreement were relatively narrow as well, which means that for probably $95 \%$ of patients, the number of cycles counted by MFES will differ from video observation by between -1.744 and 2.451 cycles. And the results also showed that the MFES was reliable in counting the number of G-R cycles, with a significant value in Spearman ranking correlation coefficient analysis. Lacking of relevant documentary evidence about the most appropriate time interval, a 1-h interval was chosen to prevent fatigue phenomenon according to Alagha M A [17]. Too short or too long would affect the results [18].

Surgery is widely recognized as the most direct way to decompress cervical spinal cord [19]. And as a widely accepted scale for the severity of cervical myelopathy, JOA scores can also be used to assess the 
surgical efficacy. In our study, the increased JOA scores and average number of cycles in postoperative suggested the recovery of spinal cord function after decompression surgery. Furthermore, significant positive correlation was found between the improvement rates of postoperative JOA scores and the preoperative number of cycles. It suggested that the better the preoperative hand function was, the better the surgical efficacy would be. Besides, the improvement of JOA scores was positively correlated with the postoperative number of cycles correspondingly increased, which might be the result of restoration of blood circulation in spinal cord after decompression surgery [20]. The fatigue phenomenon in healthy individuals and the freezing phenomenon in patients have been observed by Hosono, N. et al [2]. In our study, we found that there were significant differences between N1 and N2 in patients after surgery. The reappearance of fatigue phenomenon in postoperative indicated the recovery of impaired neurological function, which could also be a measurement for evaluating the efficacy of surgery treatment.

Compared with the original 10s G-R test, the MFES can show more details of the movements of the fingers and can provide more endpoints for the comprehensive evaluation of postoperative hand function recovery. The results showed that the waveforms of ulnar three fingers in postoperative were significantly higher and narrower than those in preoperative. And the ratios of wave height to wave width of five fingers were significantly higher in postoperative than those in preoperative. The increased wave height indicated increased range of motions of affected fingers and the declined wave width indicated increased hand dexterity of affected fingers after decompression surgery. These all indicated the recovery of spinal cord function after decompression surgery. Furthermore, MFES can simultaneously convert the movements of five fingers into waveforms, allowing direct observation of abnormal waveforms and which fingers are severely affected, and it can store every patient's data for future comparison with their own performances, allowing direct observation and objective assessment of which fingers recovered or worsened after surgery. This is an advantage of MFES compared with previous methods.

Ono [3] firstly developed the original 10s G-R test, which was simple and easy to conduct in clinical settings and its effectiveness was later demonstrated [21]. Hence, the 10s G-R test was chosen as primary movement of hands for MFES evaluating. However, it is subject to inter-observer variability and requires manual counting for subsequent analysis, which declines its practicability for clinical use. Further, visual observation and manual counting may not guarantee the standard of patients' movements and may count the incomplete flexions and extensions and then influence its accuracy. MFES addressed these issues by replacing manual counting with automatic counting, replacing human observations with characteristic waveforms. Moreover, previous performance tests had only roughly assessed hand motor function, unable to clearly record finger movements, which were inaccurate and insufficient, while the MFES firstly recorded the detailed finger movements by using virtual reality supported playback at different speeds, allowing more detailed analysis of fingers movement. In addition, some patients may try to move their fingers quickly without full flexions and/or extensions, while others may move their fingers slowly and focus more on complete flexions and extensions, which notes that using the number of cycles as the only endpoint is insufficient. However, the measurement of relevant waveform parameters (especially the ratio of wave height to wave width) contributes to eliminate such interferences and provides more endpoints for comprehensive evaluation of hand dysfunction. In the end, we have to point 
out the limitations of MFES. Firstly, it's not suitable for myelopathy patients presented only with lower limb symptoms and/or only with sensation disorders of the hand [22]. Secondly, the device looks complicated and inconvenient for clinical use. We'll simplify it further, keeping the main components (the wise-gloves and the computer with software) and removing the redundant ones (cameras); Thirdly, we only evaluated the preoperative and the postoperative hand function but without comparison data from healthy individuals. Because our main purpose in this study was to report this new assessment tool and preliminarily evaluated its value in detecting the postoperative motor function of the hand. Finally, the sample size of this study is small, and large-scale clinical evaluation is required to further analyze the complicated waveforms.

\section{Conclusions}

MFES is a reliable and valid assessment tool for myelopathy hand, which can detect small changes of neurological function after decompression surgery.

\section{Abbreviations List}

1. MFES: Myelopathy-hand Functional Evaluation System

2. CSM: Cervical Spondylotic Myelopathy;

3. JOA: Japanese Orthopedics Association;

4. ACDF: Anterior Cervical Discectomy and Fusion;

5. ACCF: Anterior Cervical Corpectomy and Fusion;

6. G-R: Grip and Release.

\section{Declarations}

\section{ETHICAL APPROVALS}

The ethics of this study was approved by the Renji Hospital Ethics Committee.

\section{SOURCE OF FUNDING}

This work was sponsored by the Program of Shanghai Subject Chief Scientist (PYIV-17-002) and the Shanghai Municipal Health Commission (2018ZHYL0215).

\section{CONFLICT OF INTEREST}

The authors declared no conflict of interest.

\section{References}


1. Fehlings M G, Tetreault L A, Wilson J R, et al. Cervical Spondylotic Myelopathy: Current State of the Art and Future Directions[J]. Spine, 2013, 38: S1-S8.

2. Hosono, N., et al. A simple performance test for quantifying the severity of cervical myelopathy. $J$ Bone Joint Surg Br, 2008, 90(9): p. 1210-3.

3. Ono K, Ebara S, Fuji T, et al. Myelopathy hand. New clinical signs of cervical cord damage[J]. Journal of Bone \& Joint Surgery, British Volume, 1987, 69(2): 215-219

4. Smith Zachary A, Barry Alexander J, Paliwal Monica et al. Assessing hand dysfunction in cervical spondylotic myelopathy. [J]. PLOS ONE, 2019, 14: e0223009.

5. Cooper PR, Epstein F. Radical resection of intramedullary spine and cord tumors in adults: recent experience in 29 patients. $J$ Neurosurg 1985, 63:492-9.

6. Yamauchi H, Hirabayashi K. Scoring system for cervical myelopathy. J Jpn Orthop Assoc 1944, 68:490-503 (in Japanese).

7. Yonenobu K, Abumi K, Nagata K, Taketomi E, Ueyama K. Interobserver and intraobserver reliability of the Japanese Orthopedic association scoring system for evaluation of cervical compression myelopathy. Spine 2001, 26:1890-4.

8. Jebsen R, Taylor N, Trieschman R. An objective and standardized test of hand function. Arch Phys Med Rehabil. 1969, 50:311-319.

9. Doita, M., et al., Evaluation of impairment of hand function in patients with cervical myelopathy. $J$ Spinal Disord Tech, 2006, 19(4): p. 276-80.

10. Oess, N.P., J. Wanek, and A. Curt, Design and evaluation of a low-cost instrumented glove for hand function assessment. J Neuroeng Rehabil, 2012, 9: p. 2.

11. Kimura, A., et al. Tally counter test as a simple and objective assessment of cervical myelopathy. Eur Spine J. 2013, 22(1): 183-188

12. Omori Masao, Shibuya Satoshi, Nakajima Tsuyoshi et al. Hand Dexterity Impairment in Patients with Cervical Myelopathy: A New Quantitative Assessment Using a Natural Prehension Movement. [J]. Behav Neurol, 2018, 2018: 5138234.

13. Akutagawa $\mathrm{T}$, Tani $\mathrm{T}$, Kida $\mathrm{K}$ et al. A new method for characterizing hand dysfunction in cervical spondylotic myelopathy: a preliminary study. [J]. Spinal Cord, 2016, 54: 221-5.

14. Lee Sunghoon I, Huang Alex, Mortazavi Bobak et al. Quantitative assessment of hand motor function in cervical spinal disorder patients using target tracking tests. [J]. J Rehabil Res Dev, 2016, 53: 10071022.

15. Kalsi-Ryan Sukhvinder, Riehm Lauren E, Tetreault Lindsay et al. Characteristics of Upper Limb Impairment Related to Degenerative Cervical Myelopathy: Development of a Sensitive Hand Assessment (Graded Redefined Assessment of Strength, Sensibility, and Prehension Version Myelopathy). [J]. Neurosurgery, 2020, 86: E292-E299.

16. Wada E, Yonenobu K, Fukui M, Hirota Y, Kikuchi S, Toyama Y, Kawakami M, Atsushi S. Grip and Release Test (10 Seconds Test) in Healthy Volunteers and Cervical Myelopathy Patients-A Simple 
Bedside Test for the Assessment of Cervical Myelopathy. Cervical Spine Research Society 2003, 112113.

17. Alagha M A, Alagha M A, Dunstan E, et al. Development of a new assessment tool for cervical myelopathy using hand-tracking sensor: Part 1: validity and reliability[J]. European Spine Journal, 2017, 26(4):1291-1297.

18. Allen MJ, Yen WM. Introduction to measurement theory. Brooks/Cole, Monterey (CA), 1979.

19. Nancy E, Epstein, Joseph A, et al. Treatment of cervical myelopathy. In: Charles R. Claerk, ed. The Cervical Spine forth edition, Philadelphia: Lippincott Williams $\square$ Wilkins, 2005:1072

20. Miwa T, Hosono N, Mukai Y, et al. Finger-tapping Motion Analysis in Cervical Myelopathy by Magnetic-Sensor Tapping Device[J]. Journal of spinal disorders \& techniques, 2013, 26: E204-E208

21. Machino Masaaki, Imagama Shiro, Ando Kei et al. Prospective Comparison of Age- and Sex-related Differences in Quantifiable 10-S Grip and Release and 10-S Step Test Results for Diagnosis of Cervical Spondylotic Myelopathy in 454 Patients with Cervical Spondylotic Myelopathy and 818 Asymptomatic Subjects. [J]. Spine, 2017, 42: 578-585.

22. Ishida K, Tani S, Enoki Y, et al. Assessment of impaired repetitive movements and starting clumsiness associated with lower limb spasticity in compression myelopathies. J Jpn Spine Res Soc; 2006, 17:97.

\section{Tables}

TABLE 1: The demographic data of included patients with CSM.

\begin{tabular}{|llll|}
\hline & Total & Male & Female \\
\hline Number & 130 & 75 & 55 \\
\hline Mean Age & $59.03 \pm 10.18$ & $59.41 \pm 10.04$ & $58.51 \pm 10.44$ \\
\hline One Level & 25 & 11 & 14 \\
\hline Two Level & 57 & 31 & 26 \\
\hline Three Level & 40 & 28 & 12 \\
\hline Four Level & 8 & 5 & 3 \\
\hline ACDF & 42 & 22 & 20 \\
\hline ACCF & 35 & 23 & 12 \\
\hline PCL & 21 & 11 & 10 \\
\hline PCLF & 32 & 19 & 13 \\
\hline
\end{tabular}


*Mean age \pm standard deviation; CSM: Cervical Spondylotic Myelopathy; ACDF: Anterior Cervical Discectomy and Fusion; ACCF: Anterior Cervical Corpectomy and Fusion; PCL: Posterior Cervical Laminoplasty; PCLF: Posterior Cervical Laminectomy and Fusion.

Table 2: Comparison of waveform parameters in preoperative and postoperative.

\begin{tabular}{|c|c|c|c|c|}
\hline & & Preoperative & postoperative & $P$ value \\
\hline & a & $13.08 \pm 1.78 \mathrm{~mm}$ & $15.19 \pm 2.04 \mathrm{~mm}$ & $P_{1} \otimes 0.0001$ \\
\hline \multirow[t]{3}{*}{ Little finger } & b & $14.57 \pm 1.33 \mathrm{~mm}$ & $12.29 \pm 1.46 \mathrm{~mm}$ & $P_{2} \llbracket 0.0001$ \\
\hline & $a / b$ & $0.899 \pm 0.18$ & $1.24 \pm 0.29$ & $P_{3} \otimes 0.0001$ \\
\hline & a & $14.49 \pm 0.75 \mathrm{~mm}$ & $16.32 \pm 1.15 \mathrm{~mm}$ & $P_{4} \otimes 0.0001$ \\
\hline \multirow[t]{3}{*}{ Ring finger } & $b$ & $14.41 \pm 1.82 \mathrm{~mm}$ & $12.24 \pm 1.32 \mathrm{~mm}$ & $P_{5} \bowtie 0.0001$ \\
\hline & $a / b$ & $1.01 \pm 0.13$ & $1.34 \pm 0.19$ & $P_{6} \otimes 0.0001$ \\
\hline & a & $15.53 \pm 2.23 \mathrm{~mm}$ & $16.48 \pm 2.30 \mathrm{~mm}$ & $P_{7}=0.0088$ \\
\hline \multirow[t]{3}{*}{ Middle finger } & $b$ & $14.54 \pm 1.81 \mathrm{~mm}$ & $12.31 \pm 1.36 \mathrm{~mm}$ & $P_{8} \bowtie 0.0001$ \\
\hline & $a / b$ & $1.07 \pm 0.17$ & $1.35 \pm 0.15$ & $P_{9} \varangle 0.0001$ \\
\hline & a & $18.53 \pm 1.25 \mathrm{~mm}$ & $18.75 \pm 1.18 \mathrm{~mm}$ & $P_{10}=0,553$ \\
\hline \multirow[t]{3}{*}{ Index finger } & $b$ & $14.48 \pm 1.76 \mathrm{~mm}$ & $12.26 \pm 1.24 \mathrm{~mm}$ & $P_{11} \otimes 0.0001$ \\
\hline & $a / b$ & $1.28 \pm 0.25$ & $1.53 \pm 0.28$ & $P_{12} \bowtie 0.0001$ \\
\hline & a & $17.76 \pm 2.15 \mathrm{~mm}$ & $17.42 \pm 2.34 \mathrm{~mm}$ & $P_{13}=0.762$ \\
\hline \multirow[t]{2}{*}{ Thumb } & b & $14.25 \pm 1.40 \mathrm{~mm}$ & $12.22 \pm 1.24 \mathrm{~mm}$ & $P_{14} \llbracket 0.0001$ \\
\hline & $a / b$ & $1.25 \pm 0.23$ & $1.43 \pm 0.28$ & $P_{15} \llbracket 0.0001$ \\
\hline
\end{tabular}

*a: Height of the waveform $(0 \leq a \leq 20 \mathrm{~mm})$; b: Width of the waveform;

\section{Figures}




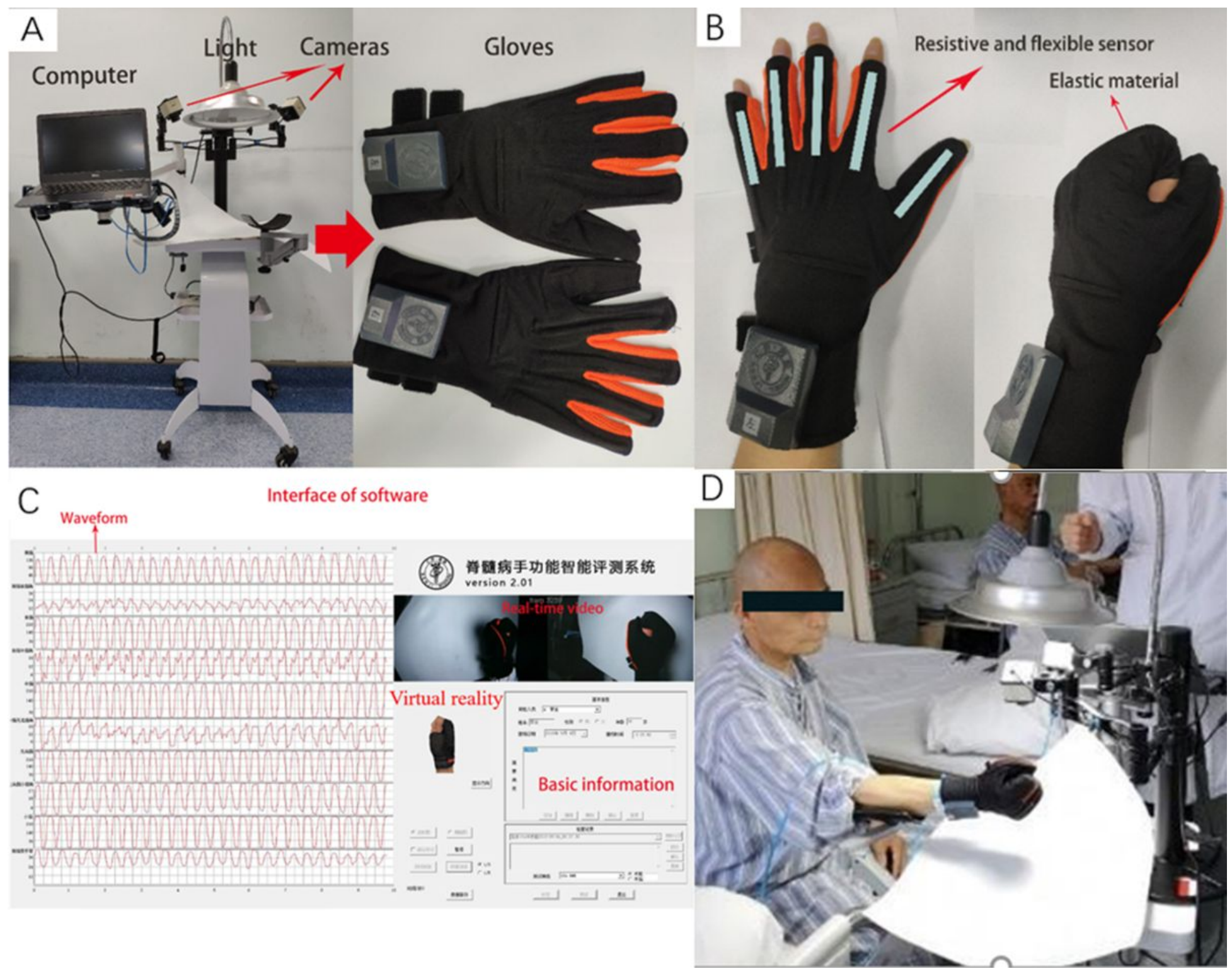

\section{Figure 1}

A: Picture showing MFES and its components. It mainly consists of a pair of gloves, a computer with a software, a light, and two mutually perpendicular cameras. B: Picture showing a pair of gloves and its components. It is made up of five resistive and flexible sensors, one transverter and elastic materials. C: The interface of software mainly consists of three modules: waveform, real-time video and basic information. D: Imaging of testing 


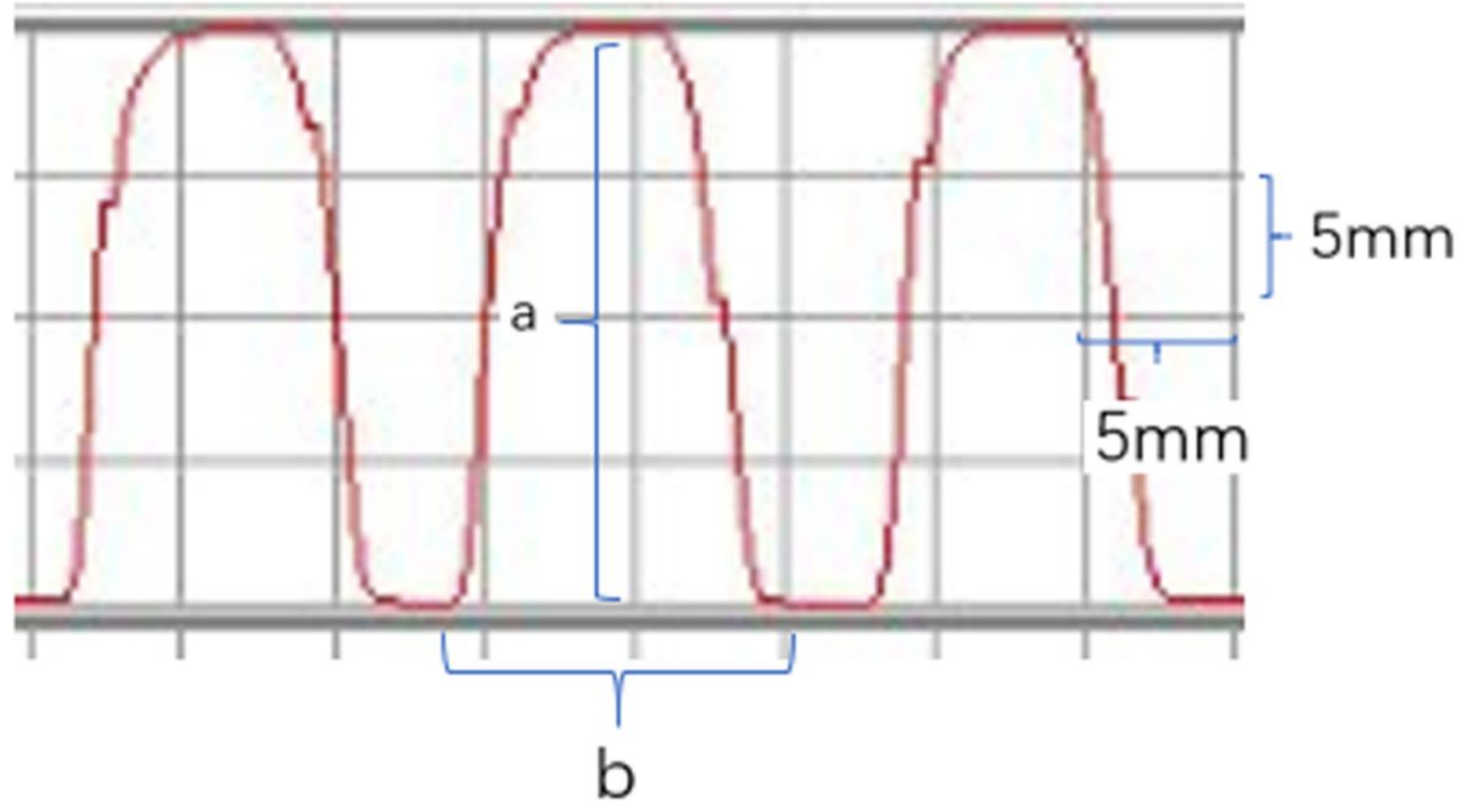

Figure 2

Measurements of relevant waveform parameters. 


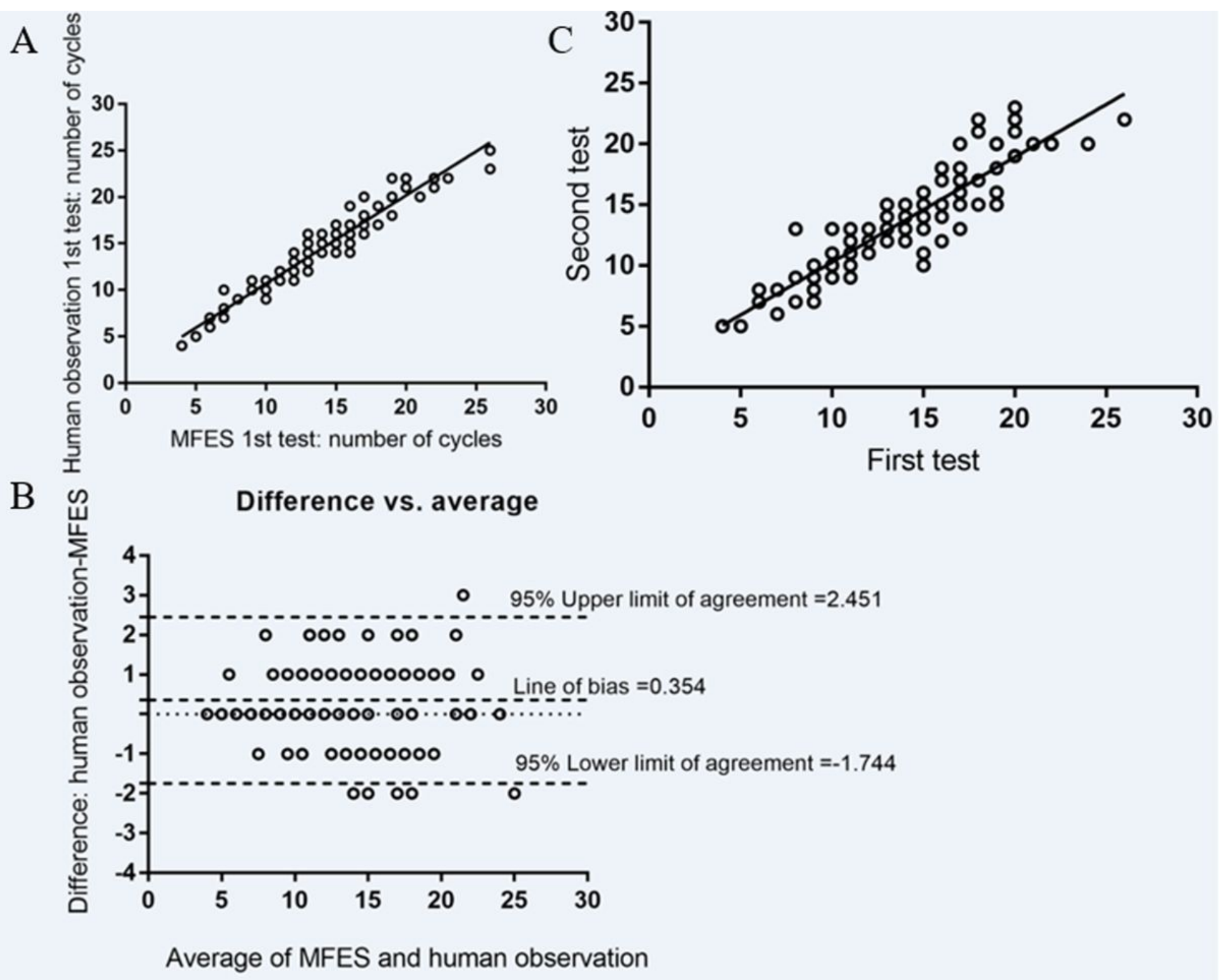

Figure 3

A: Relationship between MFES and human observations of video recordings for the number of cycles ( $r=$ $0.967, P \llbracket 0.0001)$. B: Bland-Altman plot showing the limits of agreement for the number of G-R cycles between MFES and human observations of video recordings. C: Relationship between the first 10s G-R test and the second test for the number of cycles. 
A

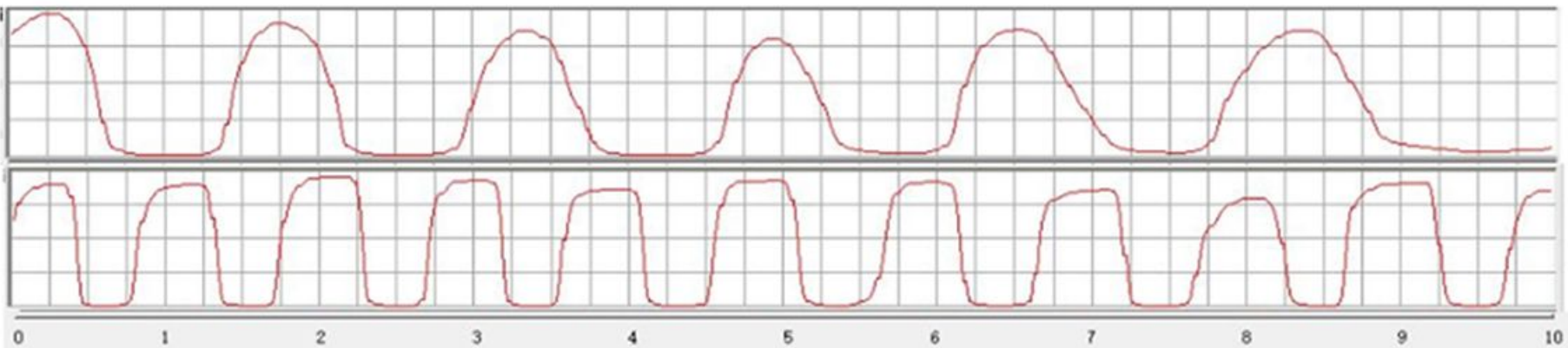

B
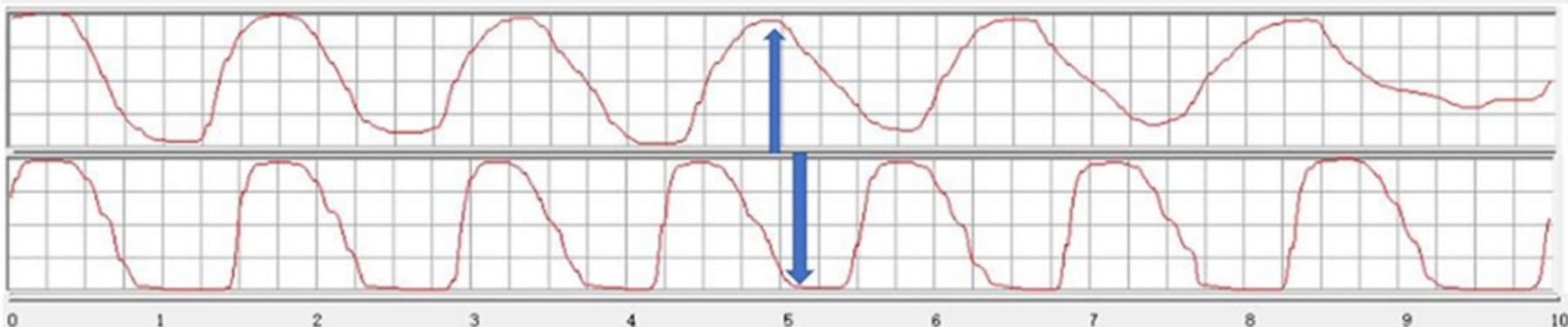

C

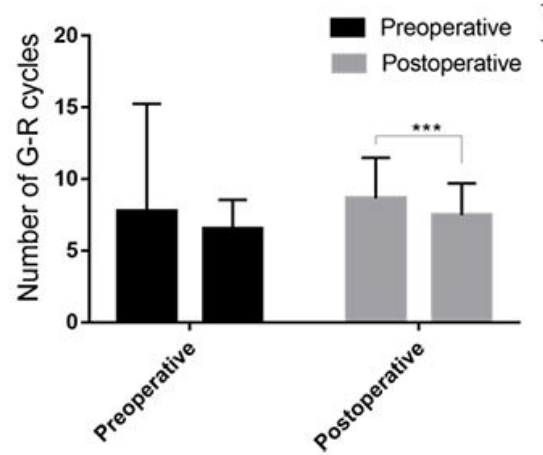

$\mathrm{D}$

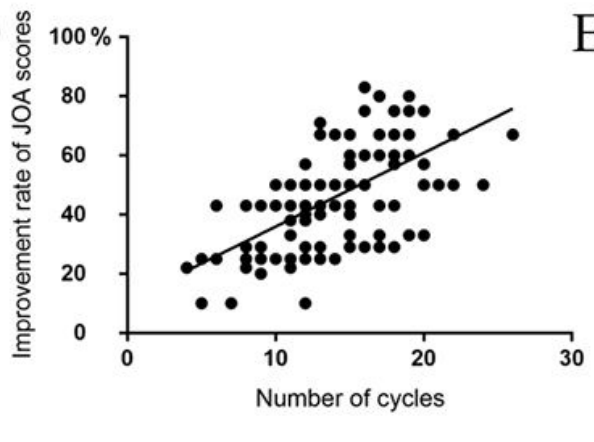

E

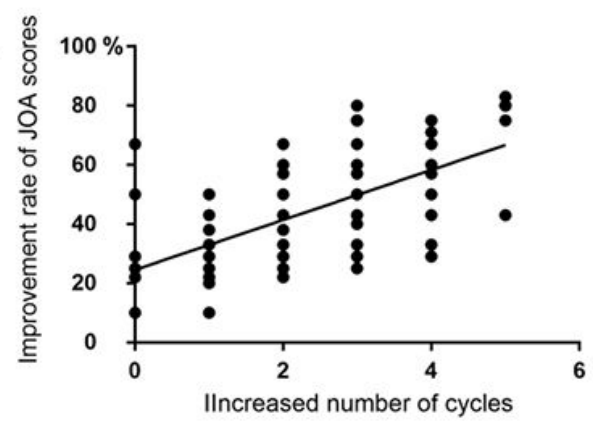

\section{Figure 4}

A: The preoperative and postoperative waveforms. B: The freezing phenomenon in preoperative and the fatigue phenomenon in postoperative. C: Comparison of $\mathrm{N} 1$ and $\mathrm{N} 2$ in preoperative and postoperative (Ppre=0.074, Ppost=0.0002). D: Correlation analysis between the number of cycles in preoperative and the improvement rates of JOA scores ( $r=0.628, P \otimes 0.0001)$. E: Correlation analysis between the improvement rates and the increased number of cycles $(r=0.585, P \otimes 0.0001)$. 


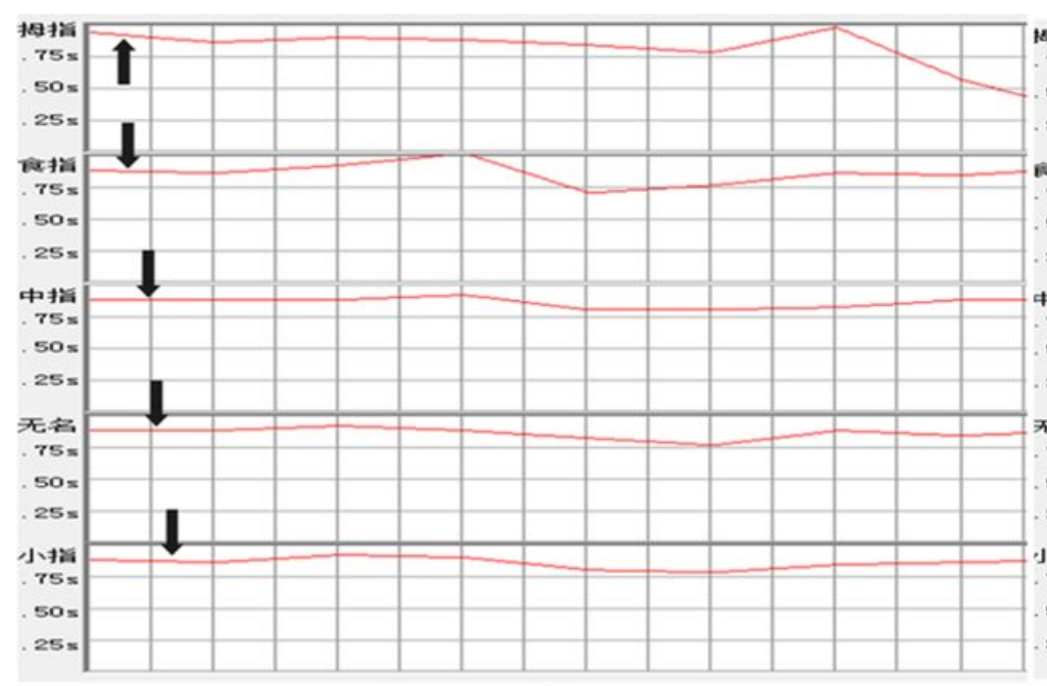

Preoperative

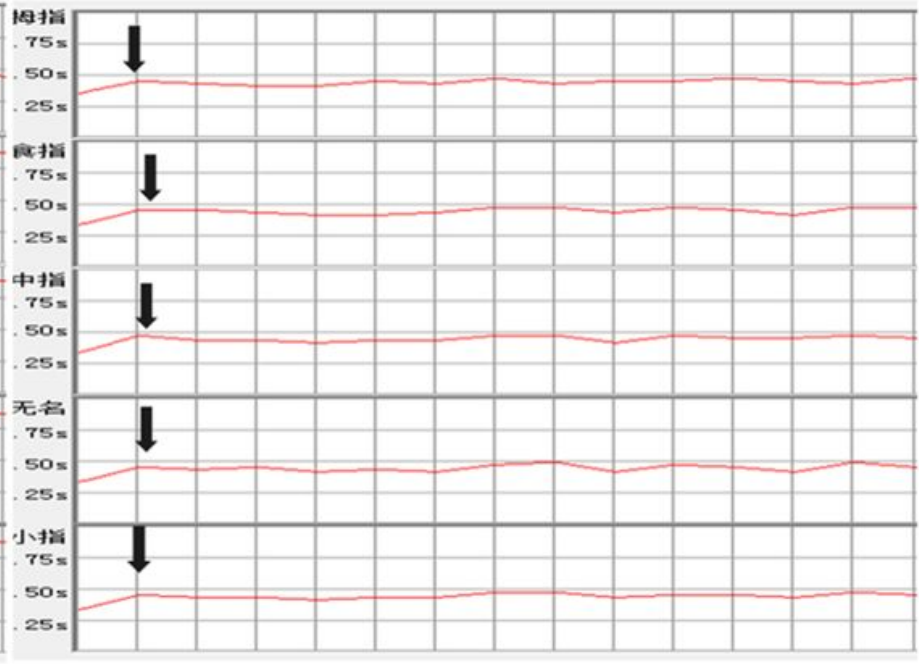

Postoperative

Figure 5

The average time per cycle in preoperative and postoperative.

\section{Supplementary Files}

This is a list of supplementary files associated with this preprint. Click to download.

- ThedetaileddesignofMFES.docx 